\title{
Impacto das Políticas de Gestão de Pessoas sobre o Desenvolvimento Profissional dos Trabalhadores
}

\author{
Ana Claudia Fernandes Monteiro ${ }^{1}$ \\ Luciana Mourão ${ }^{2}$ \\ Clarissa Pinto Pizarro de Freitas ${ }^{3}$ \\ ${ }^{l}$ Universidade Salgado de Oliveira, RJ, Brasil. \\ http://orcid.org/0000-0002-3169-9032 \\ ${ }^{2}$ Universidade Salgado de Oliveira, RJ, Brasil. \\ http://orcid.org/0000-0002-8230-3763 \\ ${ }^{3}$ Universidade Salgado de Oliveira, RJ, Brasil. \\ http://orcid.org/0000-0002-2274-8728
}

\section{$1^{\text {a }}$ Reformulação}

\section{Impacto da Percepção das Políticas de Gestão de Pessoas sobre a Percepção do Desenvolvimento}

\section{Profissional}

\section{Resumo}

O mundo globalizado demanda atuação estratégica da gestão de pessoas no ambiente laboral. O objetivo deste estudo foi investigar a influência da percepção de estratégias de gestão de pessoas sobre a percepção de desenvolvimento profissional. A amostra (de conveniência) contemplou 562 profissionais de diferentes áreas de atuação em organizações públicas e privadas, sendo que 15,4\% ocupavam cargos gerenciais. O questionário continha a Escala de Percepção Evolutiva do Desenvolvimento Profissional; e a Escala de Percepção de Políticas de Gestão de Pessoas. Os resultados da análise por equações estruturais apontaram um modelo com bom ajuste, em que a percepção de políticas que estimulam o envolvimento com o trabalho e o treinamento são preditores da percepção de desenvolvimento profissional. As conclusões corroboram a literatura da área acerca da influência das políticas de gestão de pessoas sobre as percepções dos trabalhadores e convidam à reflexão daqueles que pesquisam e atuam na área.

Palavras-chave: desenvolvimento profissional, envolvimento com o trabalho, avaliação de desempenho e competências, treinamento.

\section{Impact of Perception of Management Policies on the Perception of Professional Development}

\begin{abstract}
The globalized world demands strategic action of people management in the work environment. The objective of this study was to investigate the influence of the perception of people management strategies on the perception of professional development. The sample (of convenience) included 562 professionals from different areas of activity in public and private organizations, with $15.4 \%$ in managerial positions. The questionnaire contained the Professional Evolution Evolutionary Perception Scale and the People Management Policy
\end{abstract}


Perception Scale. The results of the analysis by structural equations pointed to a model with good adjustment, in which the perception of policies that stimulate the involvement with the work and the training are predictors of the perception of professional development. The conclusions corroborate the literature of the area about the influence of the policies of people management on the perceptions of the workers and invite the reflection of those who research and work in the area.

Keywords: professional development, involvement with work, performance and competency assessment, training.

\section{Impacto de la Percepción de las Políticas de Gestión de Personas sobre la Percepción del Desarrollo Profesional}

\section{Resumen}

El mundo globalizado demanda actuación estratégica de la gestión de personas en el ambiente laboral. El objetivo de este estudio fue investigar la influencia de la percepción de estrategias de gestión de personas sobre la percepción del desarrollo profesional. La muestra (de conveniencia) contempló 562 profesionales de diferentes áreas de actuación en organizaciones públicas y privadas, siendo que el 15,4\% ocupaba cargos gerenciales. El cuestionario contenía la Escala de Percepción Evolutiva del Desarrollo Profesional; y la Escala de Percepción de Políticas de Gestión de Personas. Los resultados del análisis por ecuaciones estructurales apuntaron un modelo con buen ajuste, en que la percepción de políticas que estimulan la participación con el trabajo y el entrenamiento son predictores de la percepción de desarrollo profesional. Las conclusiones corroboran la literatura del área acerca de la influencia de las políticas de gestión de personas sobre las percepciones de los trabajadores e invitan a la reflexión de aquellos que investigan y actúan en el área.

Palabras Clave: desarrollo profesional, implicación con el trabajo, evaluación del desempeño y habilidades, formación.

Diante do processo competitivo que transforma os produtos e serviços das organizações em commodities, as pessoas passam a ser consideradas cada vez mais um diferencial competitivo para as organizações, o que tem feito com que a área de gestão de pessoas e as suas práticas recebam mais atenção no contexto organizacional (Cleveland, Byrne, \& Cavanagh, 2015; Noe, Clarke, $\&$ Klein, 2014). Assim, o foco antes voltado quase que exclusivamente para a área financeira e para os processos operacionais, começa a voltar-se também para a gestão de pessoas em uma atuação mais estratégica e alinhada aos negócios da organização (Cleveland et al., 2015; Stone \& Deadrick, 2015).

Esse cenário impulsionou as organizações a desenvolverem políticas de gestão de pessoas que se ajustem à estratégia organizacional, valorizando os seus colaboradores (Cleveland et al., 2015; Demo, Neiva, Nunes, \& Rozzett, 2012), com o desafio de investir em mais autonomia, empoderamento e em um sistema de remuneração baseado em competências (Castro, Brito, \& Varela, 2017; Cohen, 2015).Embora esse contexto exija que as organizações tenham uma visão voltada para a gestão de pessoas, os estudos da área acerca desse tema ainda são incipientes. Assim, essa pesquisa tem como objetivo investigar a influência da percepção de estratégias de gestão de pessoas sobre a percepção de desenvolvimento profissional. Tal objetivo vai ao encontro de algumas lacunas da literatura, que sugerem o Desenvolvimento Profissional a outras variáveis do contexto organizacional (Hill, Beisiegel, \& Jacob, 2013; Monteiro \& Mourão, 2017). Além disso, o fato de utilizar uma análise confirmatória aumenta o rigor metodológico das análises de pesquisas sobre percepção de gestão de pessoas, o que também figura como uma recomendação para a área (Demo, Fogaça, Nunes, Edrei, \& Francischeto, 2011).

\section{Gestão de pessoas}

Neste trabalho considera-se que as políticas de gestão de pessoas são formadas por um conjunto de sistemas que podem ser combinados para a produção de estratégias organizacionais (Schmidt, Krawulski, \& Marcondes, 2013). Tal sistema compreende políticas voltadas para os processos de seleção e alocação de pessoas, estímulo ao envolvimento com o trabalho, treinamento e desenvolvimento de pessoas, de ava- 
liação de desempenho e competências, bem como remuneração e recompensas. Na revisão de literatura brasileira sobre políticas de gestão de pessoas realizada por Tonelli, Caldas, Lacombe \& Tinoco (2003) foi feita uma análise da produção nos principais periódicos de Administração sobre gestão de pessoas no período 1991 a 2000. Os resultados encontrados apresentam um crescimento (106\%) nas pesquisas nesse período. Entretanto, os autores alegam que esse crescimento não foi similar no rigor científico e na qualidade da produção, principalmente no que tange o ponto de vista temático, a baixa diversidade epistemológica, a frágil base metodológica e a baixíssima diversidade de origem dos autores das pesquisas.

Na década seguinte, Demo et al. (2011) realizaram uma nova revisão sobre o tema e encontraram resultados similares a Tonelli et al. (2003), no que se refere ao baixo rigor científico e qualidade da produção. $\mathrm{O}$ estudo foi realizado no período de 2000 a 2010, tendo sido identificados 109 grupos de pesquisa (8.731 pesquisadores), com maior concentração nas Ciências Sociais Aplicadas (67 grupos - 93\% da Administração) e nas Ciências Humanas (18 grupos - 44\% da Psicologia). A presença expressiva dessas duas áreas (Administração e Psicologia) sinaliza para a relação entre os componentes de gestão e os componentes do comportamento humano no trabalho. Assim, as limitações encontradas nessas revisões apontam para a necessidade de ampliação do conhecimento a respeito da gestão de pessoas.

Neste trabalho considera-se que as políticas de gestão de pessoas são formadas por um conjunto de sistemas que podem ser combinados para a produção de estratégias organizacionais (Schmidt et al., 2013). Destarte, a suposição preliminar é de que os aspectos contextuais, expressos por meio das políticas organizacionais, podem influenciar diversas variáveis de comportamento organizacional, dentre elas os resultados de desenvolvimento profissional dos empregados, uma vez que a aprendizagem no trabalho tem relação direta com o contexto (Coelho-Júnior \& Mourão, 2011; Illeris, 2007).

\section{Desenvolvimento profissional}

A aprendizagem no trabalho tem sido um tema de relevante importância para a área organizacional (Illeris, 2007; Noe et al., 2014), com uma demanda de maior atenção ao desenvolvimento das pessoas (Cleveland et al., 2015). Apesar do reconhecimento de sua importância, ainda são recentes os estudos sobre os preditores do desenvolvimento profissional (Monteiro \& Mourão, 2017), com necessidade de estudos que permitam identificar os facilitadores e os caminhos a serem percorridos para ampliar os resultados que vêm sendo obtidos (Hill et al., 2013).

Conceitualmente, o desenvolvimento profissional refere-se ao crescimento e ao amadurecimento do conhecimento, das habilidades e das atitudes do trabalhador, adquiridos ao longo da vida e resultantes de ações formais ou informais de aprendizagem no trabalho (Mourão, Porto, \& Puente-Palacios, 2014). Envolve, portanto, a construção de competências pela prática profissional e reflete-se na práxis e nas interações sociais no ambiente de trabalho, incluindo as transformações identitárias dos indivíduos e as competências coletivas desenvolvidas em equipe (Illeris, 2007; Paquay, Wouters, \& Nieuwenhoven, 2012).

A proposta de investigar a influência das percepções de políticas de gestão de pessoas sobre a percepção de desenvolvimento profissional fundamenta-se na premissa de que as políticas de gestão de pessoas assumem papel fundamental no desenvolvimento, na valorização e na retenção dos talentos (Crook, Todd, Combs, Woehr, \& Ketchen, 2011; Demo et al., 2011). Além disso, as hipóteses definidas para este estudo também se fundamentam no fato de que pesquisas na área apontam para relações positivas entre as políticas de gestão de pessoas e algumas variáveis pessoais e contextuais como: confiança impessoal e inovação organizacional (Vanhala $\&$ Ritala, 2016), valores pessoais e justiça organizacional (Fiuza, 2010), diferentes vínculos organizacionais (Pinho, Bastos, \& Rowe, 2015), satisfação no trabalho e intenção de sair da empresa por parte de diferentes gerações de empregados (Cappi \& Araujo, 2015). 
A relação entre as variáveis contextuais e a aprendizagem no trabalho, quer seja a aprendizagem formal, quer seja a informal sinaliza para a importância que as políticas organizacionais de gestão de pessoas podem ter sobre o desenvolvimento profissional de empregados (Coelho-Júnior \& Mourão, 2011). Entretanto, não foram encontrados na literatura estudos que relacionassem essas variáveis. Dessa forma, os resultados da presente pesquisa podem contribuir para o desenvolvimento de um arcabouço teórico mais consistente no que tange às relações entre essas variáveis na área da Psicologia Organizacional. Assim, foram elaboradas três hipóteses, uma para cada dimensão de gestão de pessoas.

A primeira hipótese diz respeito ao envolvimento com o trabalho, que são construções teóricas e práticas da organização, para criar um vínculo afetivo com os seus colaboradores, contribuindo para o seu bem-estar, no que se refere ao reconhecimento, relacionamento, participação e comunicação (Demo, Martins, \& Roure, 2013). O vínculo está relacionado à motivação, ao prazer e ao envolvimento com a organização, que corrobora com a noção de comprometimento encontrada nos estudos de Pinho et al. (2015). Os autores confirmam em seus estudos que estar comprometido remete a aspectos positivos, indicando a noção de envolvimento, motivação, engajamento. Além disso, os estudos sobre comprometimento apresentam relação positiva com o desempenho e o envolvimento no trabalho (Demo et. al., 2013; Singh \& Gupta, 2015) e com frequência o relacionam às variáveis de gestão de pessoas com uma expectativa de que as políticas e práticas da área possam contribuir para o desempenho organizacional (Bastos, Maia, Rodrigues, Macambira, \& Borges-Andrade, 2014). Destarte, é possível supor que trabalhadores que percebem políticas de gestão de pessoas que estimulem o envolvimento com o trabalho, tendem a ter maior percepção de desenvolvimento profissional no período de trabalho naquela organização (H1).

A segunda hipótese refere-se à outra dimensão importante na gestão de pessoas, que são as estratégias de treinamento e desenvolvimento, conceituadas como ações organizacionais que utilizam tecnologia instrucional para promover o desenvolvimento de conhecimentos, habilidades e atitudes (CHAs) para suprir lacunas de desempenho no trabalho e preparar os colaboradores para novas funções. As ações de educação e preparação para o trabalho têm um objetivo precípuo de permitir o desenvolvimento das pessoas e uma atuação mais qualificada no trabalho (Noe et al., 2014; Ribeiro \& Mourão, 2016), inclusive em termos de mudança organização, melhorias no trabalho em equipe (White, Peterschmidt, Callahan, Fitzgerald, \& Close, 2017). Nessa lógica, o suporte organizacional à aprendizagem pode resultar em mais efetividade das ações de educação corporativa e essas ações podem contribuir para uma melhoria do desempenho no trabalho em um processo cíclico e contínuo de melhoria (Bell, Tannenbaum, Ford, Noe, \& Kraiger, 2017; Langmann \& Thomas, 2017). Assim, uma vez que o desenvolvimento profissional está fortemente ancorado nas aprendizagens, nas situações de trabalho e nas oportunidades de qualificação que as pessoas têm (Haemer, Borges-Andrade, \& Cassiano, 2017; Mourão et al., 2014), supõe-se que aqueles que percebem um sistema de treinamento mais efetivo em suas organizações de trabalho, tenderão também a perceber um maior desenvolvimento profissional desde o seu ingresso em tais organizações (H2).

Em conclusão, no tocante à terceira hipótese, tem-se a avaliação de desempenho e competências, cujos modelos avaliativos variam em função das características de trabalho, objetivos, cultura e recursos de cada organização (Peixoto \& Caetano, 2013). Tal avaliação pode, por um lado, representar uma limitação quando há um desalinhamento entre as políticas de gestão de pessoas e as práticas gerenciais (Philadelpho \& Macêdo, 2007); por outro lado, pode influenciar positivamente o ciclo de desenvolvimento das organizações, sendo considerada como a base da gestão de pessoas (Brito, 2011). Assim, enquanto os dirigentes associam a avaliação de desempenho à produtividade e à competitividade da empresa no mercado, os trabalhadores 
a associam à promoção, recompensas, mobilidade interna e desenvolvimento profissional (Peixoto \& Caetano, 2013; Philadelpho \& Macêdo, 2007). Destarte, supõe-se que os trabalhadores que avaliam mais positivamente as práticas de avaliação de desempenho em sua organização, tendem a ter percepção de maior desenvolvimento profissional ao longo de sua carreira na empresa atual (H3). Considerando as hipóteses e o objetivo da pesquisa, realizou-se um estudo inferencial, de corte transversal cujo método é detalhado a seguir.

\section{Método}

\section{Participantes}

A amostra foi de conveniência e compreendeu 562 trabalhadores brasileiros, com a seguinte distribuição por regiões geográficas: Sudeste $-55,3 \% \%$; Nordeste - 17,5\%\%; Sul - 12,0\%; Centro-Oeste-11,7\%; e Norte $-3,5 \%$. Os trabalhadores pesquisados eram provenientes de organizações privadas (73,3\%), públicas (22,9\%) e do terceiro setor $(3,8 \%)$. Nessa amostra, $15,4 \%$ ocupavam cargos gerenciais. O porte das organizações variou de micro (até 10 empregados - 10,6\%) a grandes empresas (mais de 500 empregados - 39,1\%) e as organizações de pequeno porte (de 11 a 100 empregados $-25,0 \%$ ) e médio porte (de 101 a 500 empregados - 25,3\%) apresentaram percentuais similares.

Os critérios de inclusão para a participação no estudo abrangeram: a) participantes deveriam estar trabalhando há ao menos um ano na mesma organização e função; b) profissionais estarem inseridos em uma instituição pública ou privada; c) as organizações nas quais os profissionais estavam vinculados deveriam possuir políticas de gestão de pessoas; e d) os profissionais deveriam ter entre 18 a 70 anos. A adoção do critério de inclusão dos profissionais possuírem vínculo empregatício há pelo menos um ano no mesmo emprego justifica-se pela necessidade de que as pessoas tenham uma perspectiva temporal para analisar a sua percepção de desenvolvimento profissional, bem como as políticas de gestão de pessoas adotadas pela organização (Sackett, Lievens, Van Iddekinge, \& Kuncel, 2017). Como critério de exclusão da amostra foi considerada a existência de $2 \%$ ou mais dos dados em branco, o que correspondeu a 21 casos (4\% da amostra) (Cheema, 2014).

O perfil dos participantes indicou predomínio de mulheres (58,9\%), a idade variou de 18 a 64 anos, com média de 33,1 anos (Desvio-padrão=9,40). Em relação à escolaridade, predominaram o nível médio $(52,1 \%)$ e superior (43,8\%), sendo que $13,2 \%$ eram pós-graduados. O tempo de trabalho na atual organização foi heterogêneo, com amplitude amostral de 1 a 35 anos e mediana de quatro anos.

\section{Instrumentos de coleta de dados}

No presente estudo, foram utilizados um questionário sociodemográfico e laboral e dois instrumentos com evidências de validade em amostras brasileiras, sendo um para a percepção de desenvolvimento profissional e outro para a percepção das políticas de gestão de pessoas. O questionário sociodemográfico e laboral investigou as características como idade, sexo, escolaridade, além de dados laborais dos participantes (e.g., tempo de trabalho, tamanho da organização, número de colegas de trabalho).

A percepção do desenvolvimento profissional foi avaliada pela Escala de Percepção Evolutiva do Desenvolvimento Profissional (EPEDP), desenvolvida por Mourão et al. (2014), composta por 13 itens, associados a uma escala tipo Likert de cinco pontos (1 = "nenhum preparo" e 5 = "totalmente preparado"). A EPEDP pode avaliar o desenvolvimento profissional por meio de um escore geral, assim como investigar as dimensões de percepção de desenvolvimento técnico e de percepção de desenvolvimento atitudinal. A fim de compreender percepção de desenvolvimento profissional em profundidade, o presente estudo investigou as dimensões de percepção de desenvolvimento técnico, formada por oito itens da EPEDP, e percepção de desenvolvimento atitudinal, composta por quatro itens da EPEDP. Um exemplo de item: "Realizar as atividades de forma autônoma". Nessa escala 
o escore de cada item é calculado a partir da subtração dos valores das respostas relativas ao desenvolvimento profissional no momento de início da carreira daqueles valores apontados como de desenvolvimento atual, ou seja, valores atuais menos valores iniciais. Na validação original da escala o grau de precisão foi de 0,94 (Alpha de Cronbach) e no estudo atual foi de 0,92 .

Para a avaliação das políticas organizacionais na área de recursos humanos foi adotada a Escala de Percepção de Políticas de Gestão de Pessoas (Demo et al., 2012). Foram utilizadas três dimensões do instrumento, totalizando 22 itens assim distribuídos: Envolvimento (12 itens) e.g.: "A organização onde eu trabalho se preocupa com meu bem-estar"; Treinamento e Desenvolvimento (6 itens) e.g.: "Na organização onde eu trabalho, as necessidades de treinamento são levantadas periodicamente"; e Avaliação de Desempenho e Competências (5 itens) e.g.: "A organização onde eu trabalho realiza avaliações de desempenho e competências periodicamente". Os índices de consistência (Alphas de Cronbach) obtidos no estudo original e na presente pesquisa foram, respectivamente, de: $0,88 \mathrm{e}$ 0,91 para envolvimento com o trabalho; 0,88 e 0,84 para treinamento e desenvolvimento; 0,86 e 0,85 para avaliação de desempenho e competências.

\section{Procedimentos de coleta de dados}

Antes do início da coleta de dados, o projeto de pesquisa foi inscrito na Plataforma Brasil e aprovado por um comitê de ética em pesquisa da Universidade Salgado de Oliveira (CAEE 45214115.9.0000.5289). Aos participantes foi esclarecido o objetivo da pesquisa, garantido sigilo das respostas individuais, explicada a possibilidade de desistência de participação em qualquer fase do processo, bem como disponibilizado acesso aos resultados da pesquisa. Todos os participantes assinaram o Termo de Consentimento Livre e Esclarecido.

O instrumento de coleta de dados foi aplicado em todas as regiões brasileiras, com o auxílio de uma rede de aplicadores. A aplicação foi dividida em duas formas: distribuição e recolhimento dos questionários em papel (79\% da amostra), e envio do questionário a partir de um sistema on-line (21\%). As instruções de preenchimento do questionário permitiam que o instrumento fosse autoaplicável e o tempo de preenchimento era livre.

Para maior fidedignidade dos dados, foi estabelecido um sistema de acompanhamento do processo coleta de dados. A equipe de pesquisadores recebeu treinamento online e um material com procedimentos detalhados sobre a aplicação dos questionários. A cada seis pesquisadores havia um líder que recebia e conferia o preenchimento dos questionários e apresentava o relato para a coordenadora geral da pesquisa. O período de coleta foi de dez semanas e ocorreu no segundo semestre de 2016. Os pesquisadores foram instruídos a não realizar aplicações no ambiente laboral dos pesquisados, uma vez que o instrumento continha avaliações das percepções de políticas de gestão de pessoas das organizações.

\section{Procedimentos de análise de dados}

Foram realizadas análises descritivas e exploratórias, por meio do software Statistical Package for the Social Science for Windows (SPSS, versão 21.0), para investigar a presença de casos extremos, a distribuição dos casos omissos, a distribuição de frequência e os escores médios de cada variável. Também foram observadas as condições de número mínimo de dez observações por item do questionário e de pelo menos três indicadores por variável latente. A manutenção dos itens nas escalas foi condicionada à carga fatorial mínima de 0,32.

Foi observado que os valores de inflação dos fatores (variance inflation factors, VIF) das relações das três variáveis independentes estavam abaixo de 2 , indicando a ausência de multiconearidade na amostra investigada. As variáveis também cumpriram os critérios de homocedasticidade e linearidade (Hair, Black, Babin, \& Anderson, 2014). O pressuposto de normalidade não foi observado nas dimensões envolvimento com o trabalho, treinamento e desenvolvimento e avaliação de desempenho e competências, uma vez que os testes 
de Kolmogorov e Shapiro-Wilk foram estatisticamente significativos (Hair et al., 2014).

Na presente pesquisa optou-se pelo uso da modelagem das equações estruturais, pois essa se constitui como uma estratégia de análise robusta que permite investigar o modelo proposto incluindo as seis hipóteses simultaneamente. A modelagem de equações estruturais possui caráter confirmatório, sendo utilizada para investigar se as relações observadas em estudos prévios de caráter exploratório (Bastos et al., 2014; Mourão et al., 2014; Peixoto \& Caetano, 2013) são observados no modelo testado. A relevância de investigar as hipóteses simultaneamente no mesmo estudo centra-se na busca de minimizar possíveis efeitos de variáveis aleatórias e resíduos, presentes em análises que investigam as hipóteses deforma isolada (Byrne, 2000; Hair et al., 2014).

A modelagem de equações estruturais foi realizada por meio do software AMOS (versão 22). O método de estimação adotado foi o Maximum Likelihood Robust (MLR), em função da ausência de normalidade da amostra (Byrne, 2000). Após a especificação e a estimação do modelo, o seu ajuste foi avaliado por meio dos seguintes indicadores: qui-quadrado, Tucker-Lewis Index (TLI), Comparative Fit Index (CFI), Incremental Fit Index(IFI), Root Mean Square Error of Approximation (RMSEA) (Byrne, 2000).

O qui-quadrado $\left(c^{2}\right)$, como critério, não deve ser significativo, considerando-se o tamanho da amostra que induz a uma elevação desse indicador, adotou-se o critério da razão do qui-quadrado pelos graus de liberdade $(g l)$ inferior a 5,0 (Byrne, 2000; Kline, 2011). O TLI, o IFI e o CFI deveriam apresentar valor superior a 0,90 , para indicar a adequação do modelo aos dados (Kline, 2011). Enfim, estabeleceu-se o ponto de corte inferior a 0,06 do índice de parcimônia do modelo RMSEA (Byrne, 2000). Ressalta-se que esse tipo de análise insere o controle de erro de medida na própria modelagem, diminuindo a relevância da análise da consistência interna (precisão) dos instrumentos.

\section{Resultados}

A análise exploratória dos dados identificou um percentual de casos omissos inferior a $2 \%$, com caráter aleatório, sendo substituídos pela mediana (Cheema, 2014). Após as análises exploratórias, foram testadas as hipóteses para verificar o poder preditivo da percepção das políticas de gestão de pessoas sobre a percepção de desenvolvimento profissional. Como um ex post remedie para testar o commom method variance foram realizadas análises fatoriais confirmatórias (Richardson, Simmering, \& Sturman, 2009), que indicaram adequação das medidas e distinção entre os construtos que elas mensuram. Além disso, possíveis problemas de colinearidades também foram considerados na análise por equações estruturais. Os índices de adequação obtidos em cada momento, culminando no modelo estrutural que foi aceito como ajustado para os dados obtidos são apresentados na Tabela 1.

Tabela 1. Análise dos modelos e predição das variáveis

\begin{tabular}{|l|l|l|l|l|l|l|l|}
\hline \multicolumn{1}{|c|}{ Modelos } & \multicolumn{1}{|c|}{$\chi^{2}$} & \multicolumn{1}{|c|}{ gl } & \multicolumn{1}{|c|}{$\chi^{2} / \mathbf{g l}$} & \multicolumn{1}{|c|}{ IFI } & TLI & CFI & RSEA \\
\hline Modelo 0 & $2.566,6$ & 631 & 4,07 & 0,796 & 0,784 & 0,795 & $0,0,74(0,071-0,077)$ \\
\hline Modelo 1 & $1.746,8$ & 618 & 2,83 & 0,881 & 0,871 & 0,880 & $0,057(0,054-0,060)$ \\
\hline Modelo 2 & 893,3 & 370 & 2,41 & 0,926 & 0,919 & 0,926 & $0,050(0,460-0,055)$ \\
\hline Modelo 3 & 827,2 & 369 & 2,24 & 0,935 & 0,929 & 0,935 & $0,047(0,043-0,051)$ \\
\hline
\end{tabular}




\begin{tabular}{|l|l|l|l|}
\hline & \multicolumn{1}{|c|}{ B } & \multicolumn{1}{|c|}{$\boldsymbol{\beta}$} & \multicolumn{1}{|c|}{ SE } \\
\hline Envolvimento com o trabalho & 0,406 & $0,259^{* *}$ & 0,155 \\
\hline Avaliação de Desempenho & 0,273 & 0,190 & 0,146 \\
\hline Treinamento e Desenvolvimento & $-0,267$ & $-0,205^{*}$ & 0,134 \\
\hline Idade & $-0,022$ & $-0,195^{* * *}$ & 0,006 \\
\hline Tempo de Trabalho & 0,036 & $0,185^{* * *}$ & 0,010 \\
\hline
\end{tabular}

Notas. $*=p<0,05 ; * *=p<0,01 ; * * *=p<0,001 ;$ Modelo $0=$ Modelo nulo, com relações entre as variáveis fixadas em zero; Modelo 1 = Modelo teórico proposto sem qualquer ajuste; Modelo 2 = Modelo empírico ajustado; Modelo 3 = Modelo empírico final após ajustes sugeridos pelos Índices de Modificação (Modification Index).

Inicialmente foi testado o modelo nulo (M0), no qual as relações entre as variáveis são fixadas em zero. Tal modelo não se mostrou ajustado uma vez que a razão crítica e o RMSEA apresentaram valores acima dos limites aceitáveis. Esse resultado indicou a existência de alguma relação entre essas variáveis, assim, testou-se o modelo teoricamente proposto para este estudo (M1), que apontou parâmetros aceitáveis de RMSEA, e razão $c^{2} / g l$ abaixo de 5,0, CFI, IFI e TLI(Tabela 1). Apesar dos índices de ajuste adequados para o Modelo 1, as dimensões envolvimento com o trabalho, avaliação de desempenho, treinamento e desenvolvimento auxiliaram na compreensão da percepção de desenvolvimento profissional atitudinal, mas não contribuíram para explicar o fator de percepção de desenvolvimento profissional técnico.

Com base nesses resultados, um segundo modelo foi testado, investigando a contribuição das dimensões envolvimento com o trabalho, avaliação de desempenho, treinamento e desenvolvimento na explicação da percepção de desenvolvimento profissional atitudinal. $\mathrm{O}$ modelo 2 apresentou parâmetros aceitáveis de RMSEA e razão $\chi^{2} / \mathrm{df}$ abaixo de 5,0. Entretanto, o IFI, CFI e o TLI apresentaram valores marginais, não alcançando o valor de 0,90. A análise dos índices de modificação (Modification Index-MI) sugeriu relação entre os erros de dois itens da escala de envolvimento com o trabalho (item 1-“A organização onde eu trabalho acompanha a adaptação dos seus colaboradores aos seus cargos" e item 2 -“A organização onde eu trabalho se preocupa com meu bem-estar"), cujo valor de MI foi de 62,35. A análise do par de erros relacionados encontra respaldo na literatura uma vez que a adaptação aos cargos é associada ao bem-estar dos trabalhadores (Morgeson, Brannick, \& Levine, 2019).

Em razão disso, um terceiro modelo foi testado, incluindo as alterações sugeridas pelos MI. A inclusão das correlações desses pares de erros gerou um terceiro modelo (M3) com melhores parâmetros $\chi^{2} / \mathrm{df}=2,24$, $\mathrm{IFI}=0,935, \mathrm{TLI}=0,929, \mathrm{CFI}=0,935$ e RMSEA 0,047 (Tabela 1).Pontua-se que os efeitos da idade e do tempo de trabalho foram controlados na avaliação do M1, M2 e M3, sendo que a idade e o tempo de trabalho contribuíram para a explicação da percepção de desenvolvimento profissional atitudinal em todos os três modelos. No terceiro modelo pode-se observar que o avanço da idade está relacionado a menores percepções de desenvolvimento profissional atitudinal $(\beta=-0,20 ; p<0,01)$, enquanto o aumento do tempo de trabalho relaciona-se de forma positiva com a percepção de desenvolvimento profissional atitudinal $(\beta=0,19 ; p<0,01)$ (Tabela 1).

Finalmente, os resultados mostraram, que o envolvimento com o trabalho e as ações de treinamento, desenvolvimento e educação são preditores da percepção de desenvolvimento profissional atitudinal. A dimensão 
envolvimento com o trabalho emergiu do modelo como a preditora de mais relevância $(\beta=0,26 ; p<0,01)$ da percepção do desenvolvimento profissional atitudinal, de forma a confirmar a Hipótese 1 que previa uma relação positiva entre as percepções de políticas de gestão de pessoas e a percepção de desenvolvimento profissional dos trabalhadores. A direção positiva dessa relação sinaliza que quanto maior é o envolvimento do trabalho do empregado, maior tende a ser a sua percepção de desenvolvimento profissional atitudinal.

Por outro lado, a relação negativa das ações de treinamento, desenvolvimento e educação com a percepção de desenvolvimento profissional atitudinal $(\beta=$ $-0,21 ; p<0,05)$, não permitem confirmar a Hipótese 2. Ao contrário do esperado, os resultados -sugerem que quanto maior o envolvimento com as ações de capacitação agenciadas pela organização, menores os níveis de percepção de desenvolvimento profissional atitudinal.

Por fim, a Hipótese 3não foi confirmada. Tal hipótese presumia que as práticas de avaliação de desempenho e de competências influenciavam positivamente a percepção de desenvolvimento profissional ao longo da carreira. Os resultados obtidos, embora apontem um estimador positivo entre as variáveis, não foi significativo, o que impede que sejam retiradas conclusões acerca da relação entre tais variáveis.

O modelo final é apresentado na Figura 1, com os seus respectivos parâmetros padronizados e indicadores de ajuste. Em seguida é feita a discussão dos resultados.

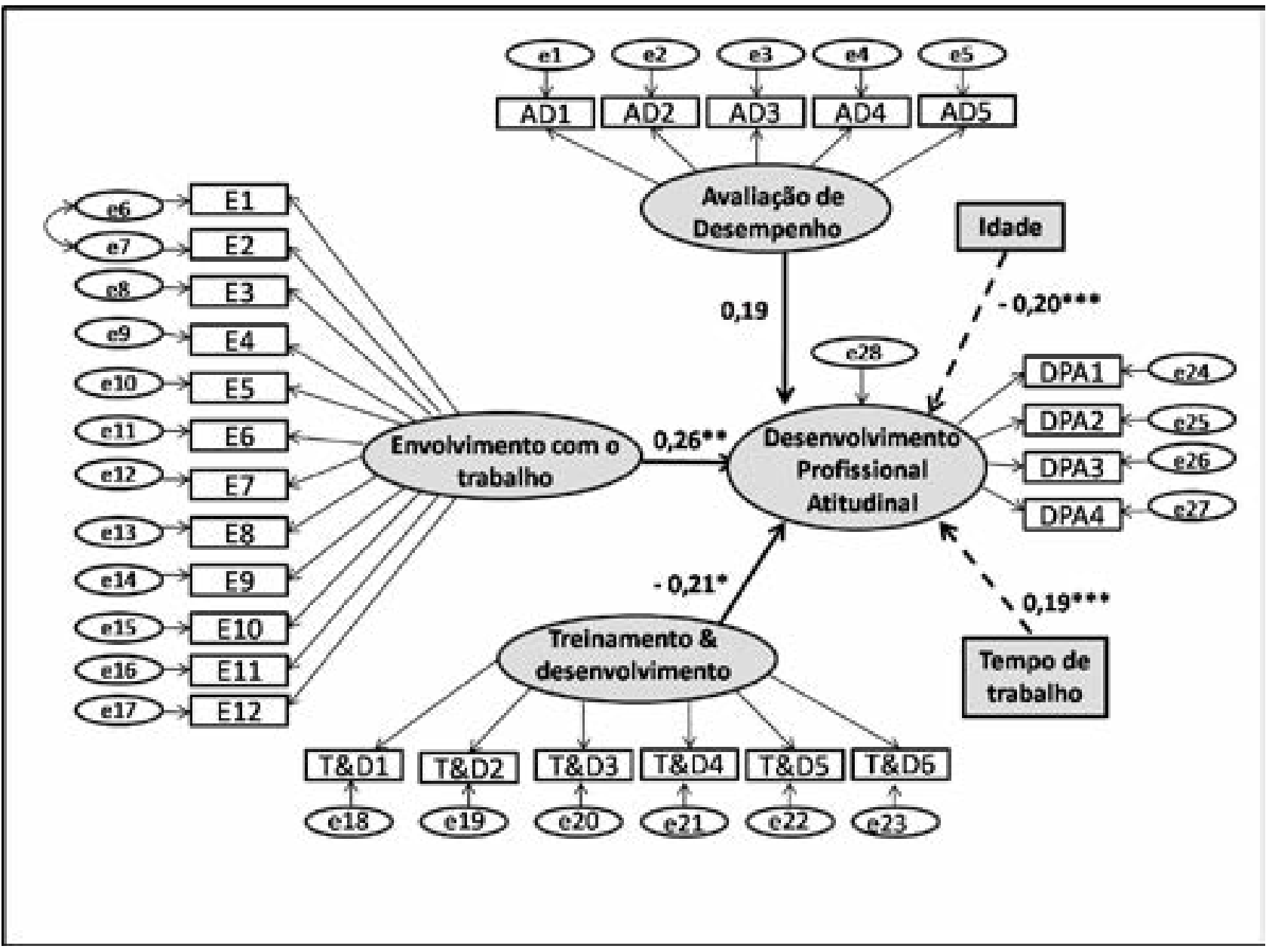

Figura 1. Modelo final da pesquisa com os parâmetros padronizados e o ajuste entre um par de itens.

Notas. ${ }^{*}=p<0,05 ; * *=p<0,01 ; * * *=p<0,001$. Linhas tracejadas indicam relação das variáveis controle com a variável dependente. 


\section{Discussão}

O presente estudo teve como objetivo investigar um modelo de predição da percepção de diferentes estratégias de gestão de pessoas (envolvimento; treinamento e desenvolvimento; e avaliação de desempenho e competências) sobre a percepção de desenvolvimento profissional. Para cumprir tal objetivo, foram elaboradas três hipóteses, discutidas a seguir.

Os resultados deste estudo apontam que trabalhadores que percebem políticas de gestão de pessoas que estimulem o envolvimento com o trabalho, tendem a ter maior percepção de desenvolvimento profissional no período de trabalho naquela organização. Apesar de não terem sido encontradas na literatura pesquisas que relacionem as políticas de envolvimento com o trabalho com a percepção de desenvolvimento profissional, os resultados de outros estudos que relacionam o envolvimento com o trabalho a outras variáveis do comportamento organizacional ajudam a explicar os resultados obtidos na presente pesquisa.

Assim, Demo et al. (2013) e Singh e Gupta (2015) relatam que a política de envolvimento é preditora do comprometimento organizacional afetivo, que por sua vez, já foi associado ao desenvolvimento profissional em estudos anteriores (Santos, 2014). Além disso, o vínculo de comprometimento está relacionado ao envolvimento com a organização, pois o estar comprometido remete a aspectos positivos, como envolvimento, motivação e engajamento (Pinho et al., 2015). Nessa lógica, o estudo confirma que pessoas que percebem mais estímulo da organização para que elas se envolvam com o trabalho, também tendem a perceber maior desenvolvimento profissional durante a sua permanência naquela organização. Em uma linha semelhante, Mourão (2018) argumenta que o desenvolvimento profissional dos trabalhadores guarda relação direta com a atuação da liderança, no sentido de os líderes motivarem o envolvimento com o trabalho dos membros de sua equipe e incentivarem processos reflexivos advindos da atuação profissional.
Outro achado desta pesquisa foi o de que a percepção de políticas de gestão de pessoas voltadas para treinamento e desenvolvimento gera um resultado negativo em termos de desenvolvimento profissional. Por um lado, os resultados obtidos neste estudo vão ao encontro dos achados de Haemer et al. (2017), que tinham encontrado relação entre o número de horas de treinamento e o desenvolvimento profissional. Por outro lado, os resultados se aproximam dos achados de Bell et al. (2017) que apontam que as ações de treinamento e desenvolvimento nem sempre surtem efeito, dependendo não apenas da existência de ações de capacitação, mas também de outras variáveis como o suporte organizacional.

Nessa lógica, o suporte organizacional aos trabalhadores pode resultar em maior efetividade das ações de educação corporativa e contribuir para uma melhoria do desempenho no trabalho (Langmann \& Thomas, 2017). Além disso, os resultados da pesquisa atual aproximam-se daquele encontrado por Ribeiro e Mourão (2016), que encontraram evidências de que muitas vezes o investimento autodidata em qualificação profissional se sobrepõe às ações de treinamento e desenvolvimento oferecidas pelas organizações, tendo as iniciativas de qualificação dos trabalhadores mais resultados para o desenvolvimento profissional do que aquelas agenciadas pelas organizações. Tais achados podem por um lado levantar reflexões sobre as necessidades de revisão no subsistema de treinamento e desenvolvimento no sentido de que ele contribua de forma mais efetiva para o desempenho dos indivíduos e também para as melhorias da equipe e para as mudanças organizacionais (Bell et al., 2017; White et al., 2017).

Em síntese, os achados da pesquisa indicaram que as práticas de avaliação de desempenho e competências não influenciam a percepção de desenvolvimento profissional ao longo da carreira. Os resultados não indicaram uma relação significativa entre essas variáveis, o que vai ao encontro do estudo qualitativo realizado por Philadelpho e Macedo (2007), que apontava descompasso entre as percepções dos diretores e gerentes e dos trabalhadores 
da área administrativa e operacional acerca do sistema de avaliação de desempenho. Para diretores e gerentes, esse era um mecanismo de aperfeiçoamento, que permitia adequar as necessidades de recursos humanos da empresa, além de ser uma forma de controle e alinhamento dos trabalhadores aos objetivos organizacionais. Para os trabalhadores, essa era uma prática formal, como uma ordem a ser cumprida, e uma forma de pressão para aumentar o desempenho, que não gera resultados, mas que pode estar associada à promoção. Em tal pesquisa foi evidenciado, portanto, que a avaliação de desempenho é usada como um instrumento de poder na gestão de pessoas, mais do que como um recurso para o desenvolvimento dos trabalhadores.

Na mesma linha, os achados de Brito (2011), a partir de um estudo multicasos em empresas do Sistema "S", mostram que a depender da forma como a avaliação de desempenho é considerada ela pode ou não contribuir para o desenvolvimento profissional dos empregados. Assim, se a empresa apresenta alto nível do ciclo de desenvolvimento, com forte influência da avaliação de desempenho, o sistema de avaliação é a base da gestão de pessoas. Mas quando a organização se caracteriza por apresentar baixo ciclo de desenvolvimento, a avaliação de desempenho é utilizada apenas como burocracia. Nesse sentido, a relação entre as políticas de avaliação de desempenho e o desenvolvimento profissional dos empregados pode ser positiva ou negativa, a depender da forma como tal sistema de avaliação se configura na empresa.

Os resultados obtidos no estudo mostram que investir em maior autonomia e empoderamento dos trabalhadores como subsídio para um sistema de gestão de pessoas baseado em competências continua sendo um desafio como foi preconizado por Cohen (2015) e por Castro et al. (2017). O fato de as percepções de políticas de gestão de pessoas voltadas para o treinamento e desenvolvimento e para a avaliação de desempenho e competências não atuarem como preditoras positivas do desenvolvimento profissional é preocupante porque revela que tais estratégias de gestão não estão assumindo o papel que delas seria esperado em termos do desenvolvimento, da valorização e da retenção dos talentos (Cappi \& Araujo, 2015; Crook et al., 2011; Demo et al., 2011). Esses resultados também colocam em xeque a perspectiva estratégica de atuação em gestão de pessoas que é discutida por Schmidt et al. (2015) e por Stone \& Deadrick (2015).

Portanto, a comparação de pesquisas anteriores com a atual leva a uma reflexão sobre até que ponto as políticas adotadas pelas organizações estão alinhadas com as reais expectativas e necessidades dos empregados, pois seria esperada maior influência de tais políticas sobre o desenvolvimento profissional. Aliás, a própria razão de existência das políticas de gestão de pessoas é o incentivo para que os trabalhadores melhorem o seu desenvolvimento e o seu desempenho, ao mesmo tempo em que se aumenta a competitividade e a produtividade organizacional (Cleveland et al., 2015).

Em uma análise geral dos resultados da presente pesquisa, conclui-se que a percepção das políticas de envolvimento e de treinamento e desenvolvimento são preditoras da percepção de desenvolvimento profissional, enquanto a percepção dos empregados acerca e da avaliação de desempenho e competências não possui relação com o seu próprio desenvolvimento.

Tais resultados suscitam, portanto, algumas conclusões e abrem novas perspectivas ao estudo das políticas de gestão de pessoas e de sua relação com o desenvolvimento profissional. Considerar os empregados como uma possível vantagem competitiva é algo discutido desde a década de 1980, contudo, esse suposto consenso acerca da importância do investimento nos trabalhadores não se reflete, necessariamente, em melhores práticas de gestão de pessoas por parte das organizações. Nesse sentido, confirma-se a existência de distância entre o discurso e as práticas organizacionais.

Em termos de implicações práticas, o estudo sinaliza para uma necessidade de que os profissionais que atuam na área de gestão de pessoas redesenhem as estratégias relacionadas à avaliação de desempenho e a treinamento e desenvolvimento, uma vez que ambas 
não apresentaram as relações esperadas com a percepção de desenvolvimento profissional dos trabalhadores. Outra implicação prática seria investir nas políticas de gestão de pessoas voltadas para o envolvimento com o trabalho, pois tal política exerce poder de predição positiva sobre o desenvolvimento profissional. Como recomendação geral do estudo, sugerem-se mudanças nas políticas de gestão de pessoas de forma a torná-las mais alinhadas às demandas dos trabalhadores, de forma a servir de um efetivo incentivo ao desenvolvimento dos mesmos, com geração de resultados para os níveis individual, de equipes e organizacional.

$O$ fato de a idade e o tempo de trabalho contribuírem para explicar a percepção de desenvolvimento profissional atitudinal também merece ser discutido. $\mathrm{O}$ uso dessas variáveis como controle evidenciou, por um lado, que o avanço da idade está relacionado a percepções mais baixas de desenvolvimento profissional atitudinal, indicando que as pessoas tendem a perceber mais avanços em termos de desenvolvimento de atitudes quando são mais jovens. Mas, por outro lado, o aumento do tempo de trabalho relaciona-se de forma positiva com a percepção de desenvolvimento profissional atitudinal, o que confirma que a vivência de experiências influencia positivamente o desenvolvimento dos trabalhadores (Paquay et al., 2012), muito possivelmente em função da aprendizagem experiencial e da reflexão proveniente da prática (Illeris, 2007; Mourão, 2018).

Em relação às contribuições teóricas da presente pesquisa, destaca-se o fato de testar um modelo ainda não encontrado na literatura nacional e estrangeira, que investigou a influência da percepção das políticas de gestão de pessoas sobre o desenvolvimento profissional. As principais contribuições teóricas referem-se ao fato de a percepção de envolvimento com o trabalho atuar como preditor positivo do desenvolvimento profissional, enquanto a percepção de uma política de treinamento mais estruturada atua como preditor negativo do desenvolvimento profissional dos trabalhadores. Assim, os resultados desse estudo confirmam que tais políticas podem influenciar, positivamente ou negativamente, o desenvolvimento profissional, o que sinaliza para a importância das estratégias de recursos humanos no desenvolvimento dos talentos. O estudo também corrobora com as lacunas das pesquisas sobre o desenvolvimento profissional, que apontam para poucos modelos de testagem de preditores do desenvolvimento profissional, especialmente em relação a variáveis contextuais. $O$ fato de o tempo de trabalho atuar como preditor positivo da percepção de desenvolvimento profissional também configura uma contribuição teórica, sobretudo por confirmar que as experiências laborais possuem papel relevante no processo de desenvolvimento dos trabalhadores.

Contudo, algumas limitações foram identificadas na presente pesquisa. Uma delas é a realização da coleta de dados em um momento único, o que pode aumentar a chance de viés nos resultados, não tendo sido adotado procedimentos ex ante que pudessem minimizar a ocorrência de problemas relativos à common method variance. Outra limitação refere-se ao fato de a amostra ser de conveniência, o que impede a generalização dos resultados obtidos. Diante dessas limitações, propõe-se uma agenda para futuras pesquisas na área.

Sugere-se a realização de estudos que continuem investigando a influência de variáveis contextuais como o desenho e as características do trabalho sobre o desenvolvimento profissional, uma vez que ainda predominam pesquisas com variáveis do nível do indivíduo. Enfim, também seriam pertinentes estudos longitudinais que permitam acompanhar, ao longo do tempo, a relação das políticas de gestão de pessoas sobre o desenvolvimento profissional, de forma a testar os resultados de ações específicas implantadas periodicamente. Seriam ainda aconselhados estudos com métodos mistos, combinando surveys e entrevistas e/ ou grupos de discussão focal para possibilitar maior entendimento acerca dos mecanismos que levam aos ganhos em termos de desenvolvimento profissional. 


\section{Referências}

Bastos, A. V. B., Maia, L. G., Rodrigues, A. C. A., Macambira, M. O., \& Borges-Andrade, J. E. (2014). Vínculos dos Indivíduos com a Organização: Análise da Produção Científica Brasileira 2000-2010. Psicologia: Teoria e Pesquisa, 30(2), 153-162. Retirado de: periodicos.unb.br/index.php/revistaptp/article/download/20945/14998. https://doi.org/10.1590/s010237722014000200004

Bell, B. S., Tannenbaum, S. I., Ford, J. K., Noe, R. A., \& Kraiger, K. (2017). 100 Years of Training and Development Research: What We Know and Where We Should Go. Journal of Applied Psychology, 102(3), 305-323. https://doi.org/10.1037/apl0000142

Brito, F. F. S. (2011). A influência da avaliação de desempenho para o desenvolvimento profissional dos colaboradores: um estudo de multicasos na Amazônia. Revista de Administração de Roraima, 1(2), 67-83. https://doi.org/10.18227/rarr.vli1.587

Byrne, B. M. (2000). Strutuctural equation modeling with AMOS. New York, London: Psychology Press Taylor, Francis Group.

Cappi, M. N., \& Araujo, B. F. V. B. (2015). Satisfação no trabalho, comprometimento organizacional e intenção de sair: um estudo entre as gerações x e y. Revista Eletrônica de Administração, 21(3), 576-600. https:// doi.org/10.1590/1413-2311.0782014.54843

Castro, A. B. C., Brito, L. M. P., \& Varela, J. H. S. (2017). A ressignificação da área de gestão de pessoas e os novos papéis das pessoas e das organizações. Holos, 4, 408-423. https://doi.org/10.15628/holos.2017.5168

Cheema, J. R. (2014). Some General Guidelines for Choosing Missing Data Handling Methods in Educational Research. Journal of Modern Applied Statistical Methods, 13(2). https://doi.org/10.22237/ jmasm/1414814520

Cleveland, J. N., Byrne, Z. S., \& Cavanagh, T. M. (2015). The future of HR is RH: Respect for humanity at work. Human Resource Management Review, 25(2), 146-161. https://doi.org/10.1016/j.hrmr.2015.01.005

Cohen, D. J. (2015). HR past, present and future: A call for consistent practices and a focus on competencies. Human Resource Management Review, 25(2), 205-215. https://doi.org/10.1016/j.hrmr.2015.01.006
Coelho-Júnior, F. A., \& Mourão, L. (2011). Suporte à aprendizagem informal no trabalho: uma proposta de articulação conceitual. Revista de Administração Mackenzie, 12(6), 224-253. https://doi.org/10.1590/ $\underline{\text { s1678-69712011000600010 }}$

Crook, T. R., Todd, S. Y., Combs, J. G., Woehr, D. J., \& Ketchen, D. J.(2011). Does human capital matter? A meta-analysis of the relationship between human capital and firm performance. Journal of Applied Psychology, 96(3), 443-456. https://doi.org/10.1037/a0022147

Demo, G., Fogaça, N., Nunes, I., Edrei, L., \& Francischeto, L. (2011). Políticas de gestão de pessoas no novo milênio: cenário dos estudos publicados nos periódicos da área de administração entre 2000 e 2010. RAM-Revista de Administração Mackenzie, 12(5), 1542. https://doi.org/10.1590/s1678-69712011000500002

Demo, G., Neiva, E. R., Nunes, I., \& Rozzett, K. (2012). Human Resources Management Policies and Practices Scale (HRMPPS): Exploratory and Confirmatory Factor Analysis. BAR-Brazilian Administration Review, 9(4), 395-420. https://doi.org/10.1590/s1807$\underline{76922012005000006}$

Demo, G., Martins, P. R., \& Roure, P. (2013). Políticas de gestão de pessoas, comprometimento organizacional e satisfação no trabalho na livraria cultura. Revista Alcance - Eletrônica, 20(02), 237-254. https:// doi.org/10.14210/alcance.v20n2.p237-254

Fiuza, G. D. (2010). Políticas de gestão de pessoas, valores pessoais e justiça organizacional. RAM-Revista de Administração Mackenzie, 11(5), 55-81. https://doi. org/10.1590/s1678-69712010000500004

Haemer, H. D., Borges-Andrade, J. E., \& Cassiano, S. K. (2017). Learning strategies at work and professional development. Journal of Workplace Learning, 29(6), 490-506. https://doi.org/10.1108/jwl-05-2016-0037

Hair, J. F., Black, W. C., Babin, B.J., \&Anderson, R. (2014).Multivariate Data Analysis ( $7^{\text {th }}$ ed.). London: Pearson.

Hill, H. C., Beisiegel, M., \& Jacob, R. (2013). Professional development research: consensus, crossroads, and challenges. Educational Researcher, 42(9) 476-487. https:// doi.org/10.3102/0013189x13512674 
Illeris, K. (2007).What do we actually mean by experiential learning? Human Resource Development Review, 6(1), 84-95. https://doi.org/10.1177/1534484306296828

Kline, R. B. (2011). Principles and practice of structural equation modeling. (3rd ed.). New York: The Guilford Press.

Langmann, S., \& Thomas, S. R. (2017). Rethinking training evaluation: omnidirectional evaluation, range of audience and intentional change. The International Journal of Human Resource Management,1-26. https:// doi.org/10.1080/09585192.2017.1303782

Monteiro, A. C. F., \& Mourão, L. (2017). Desenvolvimento profissional: a produção científica nacional e estrangeira. Revista Psicologia: Organizações e Trabalho, 17(1), 39-45. https://doi.org/10.17652/rpot/2017.1.12246

Morgeson, F. P., Brannick, M. T., \& Levine, E. L. (2019). Job and work analysis: Methods, research, and applications for human resource management. Sage Publications. https://doi.org/10.4135/9781483329505

Mourão, L. (2018). The Role of Leadership in the Professional Development of Subordinates. In S. Göker (Ed.). Leadership (pp. 123-138). London: IntechOpen.

Mourão, L., Porto, J. B., \& Puente-Palácios, K. (2014). Construção e evidências de validade de duas escalas de percepção de desenvolvimento profissional. Psico - USF, 19(1), 73-85. https://doi.org/10.1590/s1413$\underline{82712014000100008}$

Noe, R. A., Clarke, A. D. M., \& Klein, H. J. (2014). Learning in the Twenty-First Century Workplace. Annual Review Organizational Psychology and Organizational Behavior, 1(1), 245-275. https://doi. org/10.1146/annurev-orgpsych-031413-091321

Paquay, L., Wouters, P., \& Van Nieuwenhoven, C. (2012). A avaliação, freio ou alavanca do desenvolvimento profissional? In L Paquay, P. Wouters, \& C. Van Nieuwenhoven, (Orgs.), A avaliação como ferramenta de planejamento do desenvolvimento profissional de educadores (pp.13-39). Porto Alegre: Penso. https://doi. org/10.11606/d.18.2002.tde-27052004-110812

Peixoto, A. L. A., \& Caetano, A. (2013). Avaliação de desempenho. Em: L. O. Borges \& L. Mourão (Orgs.). O trabalho e as organizações: Atuações a partir da psicologia.(pp 528-556). Porto Alegre: Artmed.
Philadelpho, P. B. G., \& Macêdo, K. B. (2007). Avaliação de desempenho como um instrumento de poder na gestão de pessoas. Aletheia, 26, 27-40. Disponível em: pepsic.bvsalud.org/pdf/aletheia/n26/n26a03.pdf.

Pinho, A. P. M., Bastos, A. V. B., Rowe, D. E. O. (2015). Diferentes Vínculos Indivíduo-Organização: Explorando Seus Significados entre Gestores. Revista de Administração Contemporânea, 19(3), 288-304. https://doi.org/10.1590/1982-7849rac20151635

Ribeiro, V. V., \& Mourão, L. (2016). Percepção dos docentes do ensino fundamental sobre seu desenvolvimento profissional. Revista Brasileira de Orientação Profissional, 17(1), 55-65. Retirado de: http://pepsic. bvsalud.org/scielo.php?script=sci_arttextHYPERLINK "http://pepsic.bvsalud.org/scielo.php?script=sci_arttext\&pid=S1679-33902016000100007"\&HYPERLINK "http://pepsic.bvsalud.org/scielo.php?script=sci_arttext\&pid=S1679=33902016000100007" "pidS1679-33902016000100007

Richardson, H.A., Simmering, M.J., \& Sturman, M.C. (2009). A tale of three perspectives: Examining post hoc statistical techniques for detection and correction of common method variance. Organizational Research Methods, 12(4), 762-800. https://doi. org/10.1177/1094428109332834

Sackett, P. R., Lievens, F., Van Iddekinge, C. H., \& Kuncel, N. R. (2017). Individual Differences and Their Measurement: A Review of 100 Years of Research. Journal of Applied Psychology, 102(3), 254-273. https:// doi.org/10.1037/ap10000151

Santos, R. R. R. R. (2014). Comprometimento, entrincheiramento e percepção de desenvolvimento profissional de professores do ensino médio. (Dissertação de Mestrado). Universidade Salgado de Oliveira. Niterói. Rio de Janeiro. https://doi.org/10.11606/t.48.2006.tde08102007-204729

Schmidt, B., Krawulski, E., \& Marcondes, R. C. (2013). Psicologia e Gestão de Pessoas em Organizações de Trabalho: investigando a perspectiva estratégica de atuação. Revista de Ciências Humanas, 47(2), 344-361. https://doi.org/10.5007/2178-4582.2013v47n2p344 
Singh, A., \& Gupta, B. (2015). Job involvement, organizational commitment, professional commitment, and team commitment. A study of generational diversity. International Journal, 22(6), 1192-1211. https://doi. org/10.1108/bij-01-2014-0007

Stone, D. L., \& Deadrick, D. L. (2015). Challenges and opportunities affecting the future of human resource management. Human Resource Management Review, 25(2), 139-145. https://doi.org/10.1016/j.hrmr.2015.01.003

Tonelli, M. J., Caldas, M. P.,Lacombe, B. M. B., \& Tinoco, T. (2003). Produção acadêmica em recursos humanos no Brasil: 1991-2000. Revista de Administração Eletrônica, 43(1), 105-122. https://doi.org/10.1590/s003475902003000100011

Vanhala, M., \& Ritala, P. (2016). HRM practices, impersonal trust and organizational

Innovativeness.Journal of Managerial Psychology, 31(1), 95-109.

White, M. C., Peterschmidt, J., Callahan, J., Fitzgerald, J. E., \& Close, K. L. (2017). Interval follow up of a 4-day pilot program to implement the WHO surgical safety checklist at a Congolese hospital. Globalization and Health, 13(42), 1-9. https://doi.org/10.1186/s12992$\underline{017-0266-0}$

Recebido em: 11/1/2018.

Aprovado em: 14/8/2109.

Publicado em: $\mathrm{xx} / \mathrm{x} / \mathrm{xxxx}$

Endereço para correspondência: Luciana Mourão (Rua Marechal. Deodoro, 217 - Centro, Niterói - RJ, CEP: 24030-060; (21)2609.6764/98886.6764; mourao. luciana@gmail.com).

\section{Nome: Luciana Mourão}

E-mail: mourao.luciana@gmail.com

Titulação Acadêmica: Doutora em Psicologia pela Universidade de Brasília (UnB)

Afiliação Institucional: Professora do Pós-Graduação da Universidade Salgado de Oliveira (UNIVERSO)

\section{Nome: Ana Claudia Fernandes Monteiro}

E-mail: anamonteiropsi@gmail.com

Titulação Acadêmica: Doutora em Psicologia Social pela Universidade Salgado de Oliveira (UNIVERSO)

Afiliação Institucional: Universidade Salgado de Oliveira (UNIVERSO)

\section{Nome: Clarissa Pinto Pizarro de Freitas}

E-mail: freitas.cpp@gmail.com

Titulação Acadêmica: Doutora em Psicologia

pela Universidade Federal do Rio Grande do Sul (UFRGS)

Afiliação Institucional: Professora do Pós-Graduação da Universidade Salgado de Oliveira (UNIVERSO) 